\title{
$\alpha-N$-Heterocyclic thiosemicarbazones induce ER stress-mediated CHOP activation
}

\author{
Robert Trondl ${ }^{1}$, Christian R Kowol ${ }^{1}$, Vladimir B Arion ${ }^{1,2}$, Michael A Jakupec ${ }^{1,2}$, Bernhard K Keppler ${ }^{1,2^{*}}$ \\ From 16th Scientific Symposium of the Austrian Pharmacological Society (APHAR) \\ Vienna, Austria. 25-27 November 2010
}

\begin{abstract}
Background
Blocking of DNA synthesis through inhibition of ribonucleotide reductase has been proposed to be the main mechanism of anti-neoplastic action for $\alpha-N$-heterocyclic thiosemicarbazones. Currently the best-studied agent of this class of compounds is triapine (3-amino-2-carboxaldehyde thiosemicarbazone), which has been tested in several phase I and II clinical trials. We synthesized triapine $\left(\mathrm{HL}^{\mathrm{A}}\right)$ and the corresponding terminally $N^{4}$ dimethylated derivative, 3-aminopyridine-2-carbaldehyde $\mathrm{N}, \mathrm{N}$-dimethylthiosemicarbazone $\left(\mathrm{HL}^{\mathrm{B}}\right)$. Previously, we have shown that dimethylation of the terminal amino group leads to significant amplification of the activity in cytotoxicity assays. Previously, we also discovered intrinsic fluorescence properties for both compounds. Here we present a study of intracellular distribution of the compounds and a possible new mechanism of action for $\alpha$ - $N$-heterocyclic thiosemicarbazones by induction of endoplasmic reticulum (ER) stress.
\end{abstract}

\section{Methods and results}

Fluorescence microscopy was performed on living SW480 cells (colon carcinoma) treated with $\mathrm{HL}^{\mathrm{A}}$ and $\mathrm{HL}^{\mathrm{B}}$. Microscopy images show a strong affinity to the nuclear membrane and to cytosolic structures. Co-localization studies revealed both agents are associated with structures of ER and mitochondria and co-staining images suggest an involvement of ER in its mechanism of action. SW480 cells were treated for $15 \mathrm{~h}$ with the compounds in micromolar concentrations and immunoblotting analyses were performed, resulting in high protein levels of the ER stress-mediated C/EBP homologous protein (CHOP). CHOP is known to be transcriptional activated when functions of the ER are severely impaired and is associated with mitochondria mediated apoptosis pathway. The cytotoxic potencies of $\mathrm{HL}^{\mathrm{A}}$ and $\mathrm{HL}^{\mathrm{B}}$ were determined in SW480 (colon carcinoma) and $41 \mathrm{M}$ (ovarian carcinoma) cells by means of the colorimetric MTT assay. The $\mathrm{IC}_{50}$ value of triapine $\left(\mathrm{HL}^{\mathrm{A}}\right)$ is $0.55 \pm$ $0.2 \mu \mathrm{M}$ in SW480 cells and $0.45 \pm 0.03 \mu \mathrm{M}$ in the $41 \mathrm{M}$ cell line. $\mathrm{HL}^{\mathrm{B}}$ showed $\mathrm{IC}_{50}$ values of $0.33 \pm 0.02 \mu \mathrm{M}$ in SW480 and $0.21 \pm 0.13 \mu \mathrm{M}$ in $41 \mathrm{M}$ cells, respectively. Comparing triapine with its $N^{4}$-dimethylated derivative, a 1.6-2.1-fold higher activity was observed.

\section{Conclusions}

Our results suggest that $\alpha-N$-heterocyclic thiosemicarbazones induce ER stress-mediated CHOP activation and subsequent apoptosis signaling, which is a novel mechanism of action for this class of compounds. Further investigations will help to clarify in detail the role of ER stress induction in the mode of action.

\section{Author details \\ ${ }^{1}$ Institute of Inorganic Chemistry, University of Vienna, 1090 Vienna, Austria. ${ }^{2}$ Research Platform "Translational Cancer Therapy Research", University of} Vienna, 1090 Vienna, Austria.

Published: 16 November 2010

doi:10.1186/1471-2210-10-S1-A20

Cite this article as: Trondl et al:: $\alpha-N$-Heterocyclic thiosemicarbazones induce ER stress-mediated CHOP activation. BMC Pharmacology 201010 (Suppl 1):A20. 